\title{
THE EFFICIENCY OF U-70 SCRAPER SYSTEM
}

\section{I.A. Yazynin, I.I. Degtyarev, Institute for High Energy Physics, Protvino, Moscow region, 142284, Russia}

\begin{abstract}
:
One of the main parameters of quality the scraper or collimation systems is minimizing the yield of primary particles from system in cyclic and linear accelerators. From this viewpoint in paper the choice of materials the target, scraper and collimators, and its geometrical sizes is considered taking into account the energy and parameters of formed beam.
\end{abstract}

\section{INTRODUCTION}

The collimation system in modern accelerators serves for localization of beam particles, which can be lost in the beamline or accelerator circle, in one or some specially equipped places. Basic problems solved at creation of such systems in high intensity accelerators are preventing destruction of the system elements and minimization of the output particles flux in a circle [1-3]. In paper on an example of U-70 the approach to the decision of a minimization task of an output the scattered protons is considered at use scrapers from different materials, additional scattering targets and collimators depending on the beam parameters. The calculations were made by coupled SCRAPER - RTS\&T code system [4] which modeling the particle tracing in the accelerator magnetic structure and substances of the elements taking into account a real processes of particle interactions with matter.

\section{STRATEGY OF SCRAPING}

The impact parameter of a particles on the scraper in the cyclical accelerator as a result of a beam guidance by bump-magnets or its swelling it is usual small - some microns [5]. With the help of a scattering target it is possible considerably to increase the beam impact parameter and such way to reduce the output flux from scraper, but thus there is an additional source of dangerous particles - target. On Fig.1 the scraper system layout is represented.

The particles, getting on the scraper or target, are absorbed or are scattered on some angle with reducing of energy. At small scattering they through some turns can put again on scraper (1, Fig.1), or to be lost in a circle, if their amplitude is more than the aperture of the accelerator (2), and at significant scattering to put on a system's shield (3). Therefore the most dangerous is second fraction of the scattered particles. For its reduction it is possible to use additional collimators placed by the scraper on a beam course, which together form a so-called two-stage scraper system.
As materials of the system elements the radiation stable substances having a different density and, hence, the different parameters from a viewpoint of leakage and absorption of particles - nuclear and radiation lengths were considered.

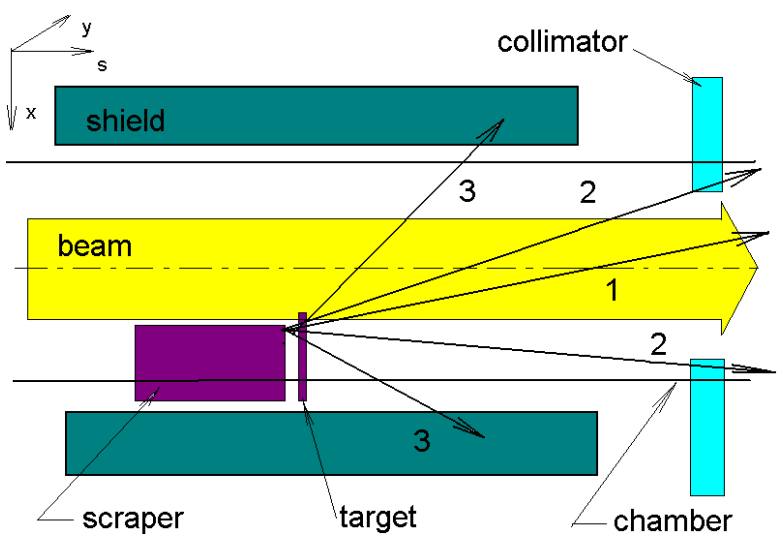

Figure 1: The scraper system layout.

\section{SINGLE TRACING}

The research of angular scattering of particles was made at hit them on scraper of large length that is practically without output of primary particles from a back face, with different impact parameters and energies.

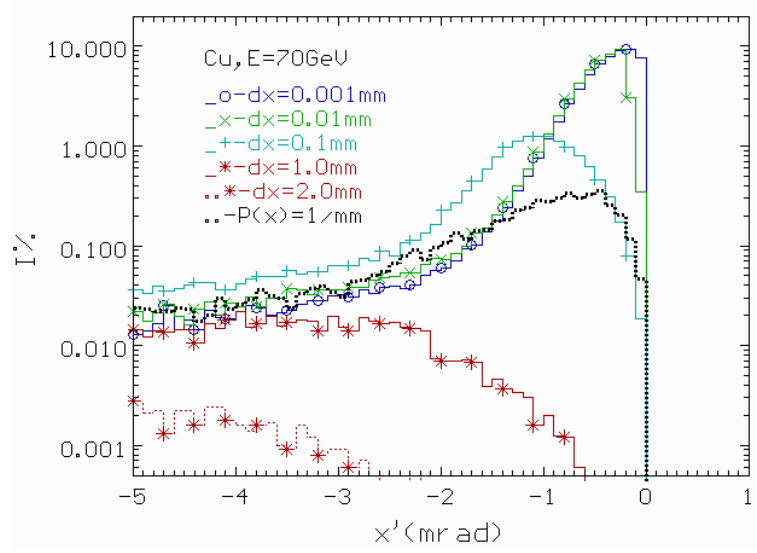

Figure 2: Angular distributions of particles outgoing from the $\mathrm{Cu}$ collimator or scraper $\left(E_{0}=70 \mathrm{GeV}\right)$.

On Fig.2 the angular normalized distributions of the scattered protons are presented at single tracing through a copper collimator at energy $70 \mathrm{GeV}$, from which it is 
visible, that the main contribution to a stream of outgoing particles in the most dangerous range of angles (for U-70

$\alpha \sim 2 \mathrm{mrad}$ ) is brought by input particles with defined impact parameter $(\mathrm{dx} \sim 0.01-0.5 \mathrm{~mm})$. The intensity of output flux strongly decreases with increase of a beam impact parameter and energy. For example, for copper the protons displaced on magnitude $\mathrm{dx}>2 \mathrm{~mm}$ practically do not bring in the contribution to an output flux of dangerous particles. Therefore at large impact parameters on scraper or collimator the density of leakage particles will be proportional to a density of particles on it edge $P(\mathrm{x})$ and, hence, is inversely proportional to size of a beam impact parameter.

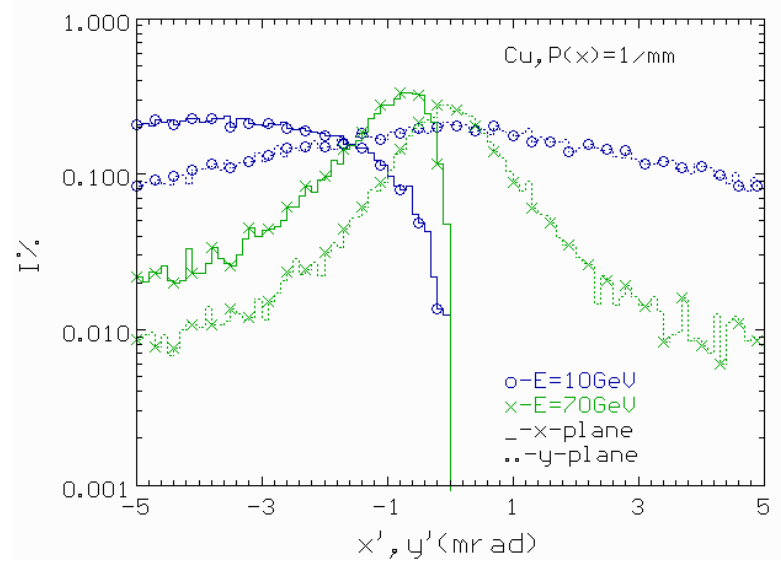

Figure 3: Angular distributions of the scattered particles.

Therefore for an estimation of collimator's efficiency the output distributions are considered at uniform input distribution of particles $P(\mathrm{x})=1 / \mathrm{mm}$. On Fig. 3 the angular distributions of the scattered particles for two energies 10 and $70 \mathrm{GeV}$ are submitted, from which it is visible, that the density of particles $\rho\left(\mathrm{y}^{\prime}\right)=0.01 \times I\left(\mathrm{y}^{\prime}\right) / \Delta \mathrm{y}^{\prime}$ in the plane perpendicular the output one is less than $\rho\left(x^{\prime}\right)$ in the range of considered angles. Therefore further in basic the attention to scattering of particles in output plane $(\mathrm{x})$ is given.

The density distributions of the scattered protons versus initial energy outgoing under the angle $\alpha=$ $2 \mathrm{mrad}$ from collimators manufactured from different materials are shown on the Fig.4. From which it is visible, that in considered energy range and scattering angles are preferable the $\mathrm{Cu}$ collimators.

Generally at design of a channel's collimation, where there is a single tracing beam, from the parameters of a beam in the channel it is possible to define optimum site and displacement of main and additional collimator's jaws and to estimate what material is more acceptable for their creation from a viewpoint of minimization losses. For example, as it is visible from the Fig.4, at the energy larger then $40 \mathrm{GeV}$ for a smaller leakage of scattering particles the light materials, and for smaller energies hard one are preferable at the sufficient accelerator aperture.

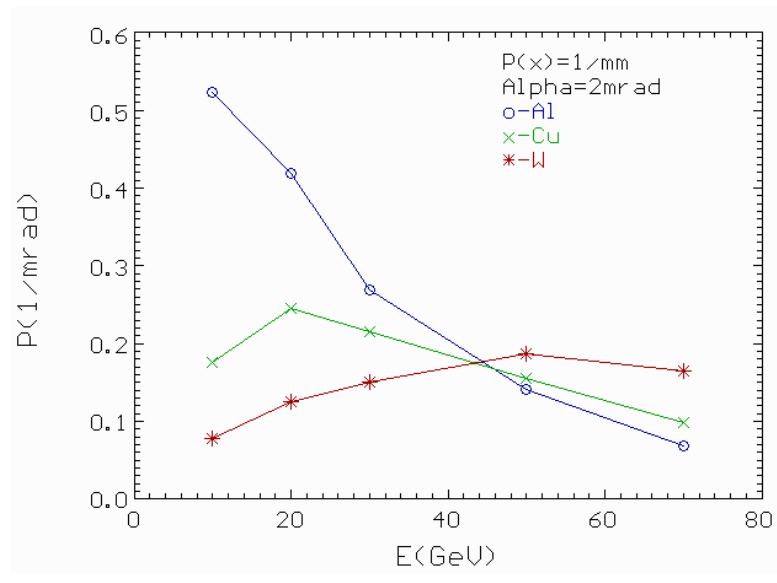

Figure 4: Density angular distributions of the scattered particles versus energy.

In collimators from light materials an integrated leakage of the scattered particles more, but they are deflected on smaller angles, and the amount of loosed particles can decrease.

\section{MULTI-TURN TRACING.}

At multi-turn tracing the form of angular distribution of particles will modify as a result of multiple passing of particles through the scraper system. To define the losses in the ring it is convenient to consider the distribution of outgoing particles on maximum amplitudes, which they can reach

$$
A=2|a| \sqrt{\beta_{m}}+\eta_{m} \times \Delta P / P
$$

Because

$$
2|a| \approx x^{\prime} / \sqrt{\beta_{0}}
$$

then $A \approx x^{\prime} \sqrt{\beta_{0} \beta_{m}}+\eta_{m} \times \Delta P / P$ where $\beta_{0}$ and $\beta_{\mathrm{m}}$ are amplitude functions in an installation site of the scraper or target and narrowest place in a ring, $\eta_{\mathrm{m}}$ is dispersion and $\Delta P$ is momentum deviation.

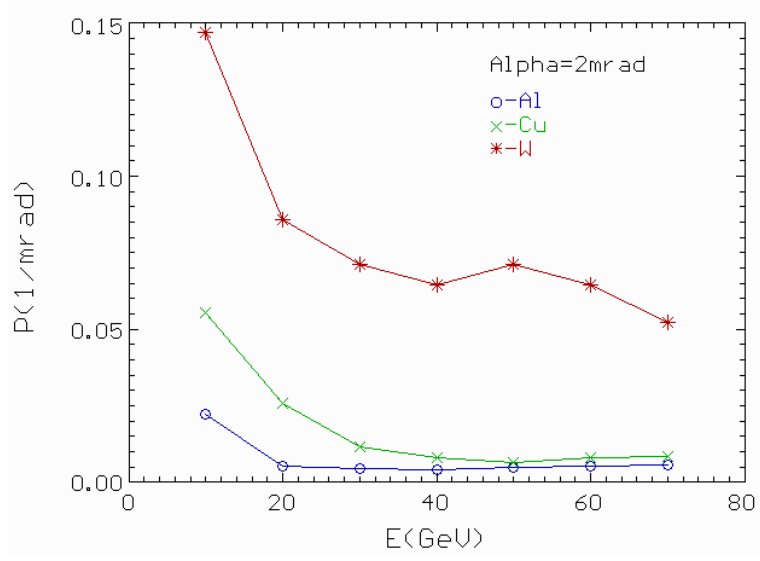

Figure 5: Density angular distributions of the scattered particles versus energy in multi-turn case. 
On the Fig.5 the density angular distributions of the scattered particles outgoing from the scraper manufactured from different materials versus energy are shown. The parameters for calculations were: frequency of betatron oscillations $Q_{\mathrm{x}}=n+0.72$, where $\mathrm{n}$ is integer, $\beta_{0}=\beta_{\mathrm{m}}=25 \mathrm{~m}, \eta_{\mathrm{m}}=2 \mathrm{~m}$. We can see the huge changing in behavior of curves in single and multi -turn scattering case (Fig.4 and 5). Most dangerous with a viewpoint of the accelerator's irradiation are the scattered particles with amplitude a little bit greater then $A_{\max }$. If to assume, that in a ring the protons with amplitudes from $A_{\max }$ up to $A_{\max }+\triangle A$ are lost, the amount of the losses will be $N=\Delta A \times P\left(x^{\prime}\right) / \sqrt{\beta_{0} \beta_{m}}$. Therefore quality of a system can be estimated on an angular density $P\left(x^{\prime}\right) / \sqrt{\beta_{0}}$ of particles scattered on an angle $\alpha \approx A_{\max } / \sqrt{\beta_{0} \beta_{m}} \sim 2 \mathrm{mrad}$, if $A_{\max }$ is $\sim 50 \mathrm{~mm}$. It is visible, that for such apertures as in U-70 that the light materials as $A l$ are more preferable in energy range up to $70 \mathrm{GeV}$.

With increase $\beta_{0}$ the amplitude of leakage particles is increased and therefore the losses grow, but also is enlaged the impact parameter on scraper, that decreases its heat load and modifies distribution of outgoing particles. The change of a relative density of losses $P\left(x^{\prime}\right) / \sqrt{\beta_{0}}$ versus amplitude function in an installation site of scraper is shown on the Fig.6, from which it is visible, that for small energy $10 \mathrm{GeV}$ the density of losses is decreases, and at bigger energy (E $\sim 40-70 \mathrm{GeV}$ ) its grows with enlaging $\beta_{0}$. That is from a viewpoint of decreasing radiation of the accelerator ring to increasing amplitude function in a scraper's installation site it is not meaningful.

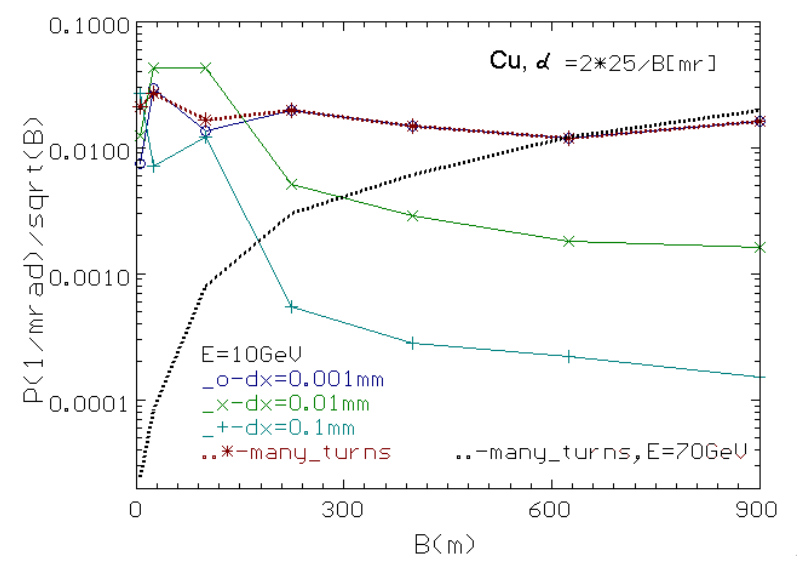

Figure 6: Density of losses versus $\beta_{0}$.

As a rule, the first collimators install on a beam course near the scraper, with the purpose of interception the particles with the large amplitudes of betatron oscillations. Even if the aperture of collimators will be smaller than the accelerator aperture $\mathrm{X}^{\prime}<A_{\max } / \beta$ all the same some fraction of the scattered particles will be lost outside of a system because of presence the momentum deviation. On Fig.7 the numerical experiment by definition of losses depending on the aperture of collimators is submitted, from which the advantage of copper scraper is visible.

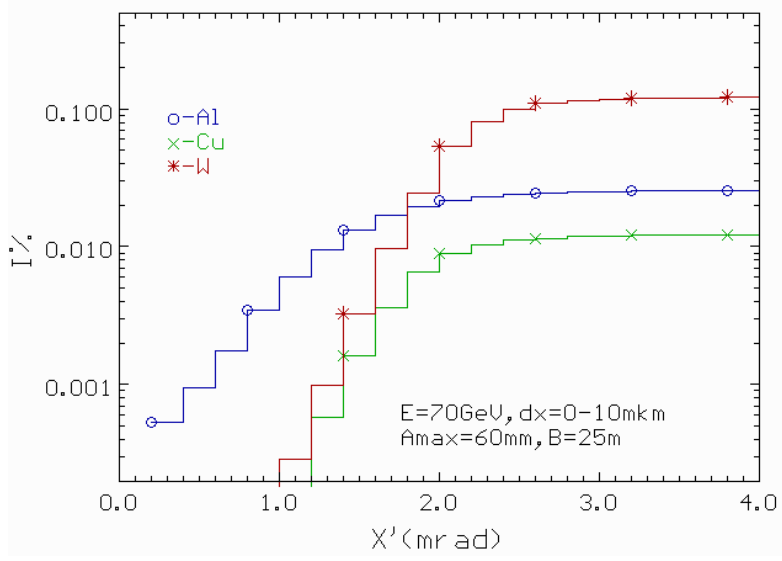

Figure 7: Losses versus the angle position of the collimator's jaws $\left(A_{\max }=60 \mathrm{~mm}, E_{0}=70 \mathrm{GeV}\right)$.

As have shown calculations the use of an addition scattering target gives an advantage in this case only at energy $<40 \mathrm{GeV}$, and at large one the system without a target is more effective. Significant reduction of an output flux from a system with increase of energy and strong dependence of leakage particles from a system versus frequency of betatron oscillations also is observed. Near to the integer and half-integer resonances the substantial growth of a density of outgoing particles is observed, that is the system efficiency reduce. The ranges of working frequencies $0.3-0.4$ and $0.6-0.9$ correspond to a minimum output of particles from a system.

\section{REFERENCES}

[1] L.Burnod, J.B.Jeanneret, "Beam losses and collimation in the LHC: A quantitative approach", CERN SL/91-39 (EA), LHC Note 167.

[2] I.I.Degtyarev, A.E.Lokhovitskii, Yu.S.Fedotov, I.A.Yazynin, "Substance choice of the scraper system elements", PAC97.

[3] I.A. Yazynin, I.I. Degtyarev, A.E. Lokhovitskii, "Efficiency of the UNK scraper system", PAC97.

[4] I.I.Degtyarev, A.E.Lokhovitskii, Yu.S.Fedotov, I.A.Yazynin, "Beam losses simulation in accelerators with extended version of the RTS\&T code", Paper presented at 17 International Conference HEACC, Dubna, 1998.

[5] I.A.Yazynin, "The impact parameter of the particles at the scraper and ways to increase it", PAC94. 